\title{
Large vessel occlusive disease associated with CREST syndrome and scleroderma
}

\author{
Peter Youssef, Helen Englert, James Bertouch
}

\begin{abstract}
Objectives-To report the cases of three patients with CREST syndrome and one patient with diffuse scleroderma who had severe macrovascular disease and only minimal vascular risk factors.

Methods-The medical histories, physical examinations, and results of clinical investigations were reviewed in four patients.

Results-These four patients had severe morbidity from macrovascular disease of the arms and legs in the presence of minimal underlying vascular risk factors. These patients represent $11 \%$ of the women with scleroderma seen at our hospital since 1974. This is a greater than threefold increase above the expected proportion of symptomatic vascular disease seen in population studies. In the patients with CREST syndrome, large vessel disease was first seen more than 10 years after the onset of Raynaud's phenomenon, which was the first manifestation of the disease. A pathological specimen of the ulnar artery from one patient showed severe luminal narrowing by an acellular material with no evidence of atheroma. Conclusions-These cases suggest an association of both the CREST syndrome and scleroderma with macrovascular disease.
\end{abstract}

(Ann Rheum Dis 1993; 52: 464-466)

Although the aetiology of scleroderma is unknown, abnormalities of the microvasculature have been implicated..$^{1-3}$ In an extensive review Norton and Nardo ${ }^{1}$ state that the frequency of vascular abnormalities is inversely proportional to the size of the blood vessels studied and that large artery disease is extremely rare.

We report the cases of three patients with CREST syndrome and one with diffuse scleroderma in whom severe macrovascular disease developed in the absence of significant vascular risk factors.

\section{Case reports}

CASE 1

A non-smoking woman with no known vascular risk factors initially presented at the age of 66 years with recurrent bilateral foot ulceration and bilaterally absent lower leg pulses below the knees. The ulcers resolved after bilateral lumbar sympathectomies.
Three years later gangrene of her right foot and right index finger developed, requiring amputation below the right knee and amputation of the finger. Further questioning revealed a history of Raynaud's phenomenon and oesophageal reflux symptoms which had been present for more than 10 years, and physical examination revealed sclerodactyly and telangiectasia on the face and hands. Anticentromere antibodies were present at a titre of $1 / 2560$. A diagnosis of CREST syndrome was made.

At the age of 74 years gangrene of the left foot developed. Femoral angiography showed severe narrowing of the left anterior tibial artery and patent superficial and deep femoral and popliteal arteries. The discrepancy in the extent of vascular disease below and above the knees was atypical of atherosclerotic peripheral vascular disease. Blood glucose and lipid levels were normal. The activated partial thromboplastin time was not prolonged and anticardiolipin antibodies were not detected. Amputation of the left foot was initially performed, but was later extended to below the knee.

Currently the only clinical problems are intermittent Raynaud's phenomenon and an oesophageal stricture. There has been no further gangrene of the limbs.

CASE 2

A non-smoking woman with no known vascular risk factors presented at the age of 75 years with digital pulp ischaemia of both hands. A diagnosis of CREST syndrome was made because of a long history of Raynaud's phenomenon and oesophageal reflux symptoms, and the presence of digital telangiectasia and sclerodactyly. Anticentromere antibodies at a titre of $1 / 5120$ were detected. Resolution of the ischaemia followed treatment with guanethidine blocks to her arms and nifedipine by mouth.

Six months later bilateral calf claudication developed, more severely on the right leg. Femoral angiography showed widespread arterial narrowing in the legs and severe right superficial femoral artery stenosis at the adductor hiatus. Blood glucose and lipid levels were normal. The activated partial thromboplastin time was not prolonged and anticardiolipin antibodies were absent.

Angioplasty was performed on the right superficial femoral artery with improvement in symptomatology of the right calf. Bilateral calf claudication continues to limit daily activities despite treatment with nifedipine. 
CASE 3

A woman presented at the age of 67 years with ischaemic lesions of the left heel, left fourth and right second fingers, and bilateral claudication of her legs. Arterial Doppler studies showed occlusion of the left ulnar and right radial arteries with normal proximal pulses. Successful treatment with guanethidine blocks and intra-arterial reserpine to her arms was instituted.

A diagnosis of CREST syndrome was made because of a history of Raynaud's phenomenon and the presence of sclerodactyly and digital telangiectasia. Anticentromere antibodies were found at a titre of $1 / 2560$ and a barium swallow test showed an oesophageal motility disorder. The patient was a lifetime non-smoker and had mild hypertension requiring no treatment. Between the ages of 63 and 66 years she had three uncomplicated myocardial infarcts with no evidence of regional wall abnormalities on echocardiography. Coronary angiography was not performed. Blood glucose and lipid levels were normal. The activated partial thromboplastin time was not prolonged and no anticardiolipin antibodies were detected.

Three months after the initial presentation there was increasing claudication of the left leg and progressive ischaemia of the right index finger. Femoral angiography showed severe arterial occlusion in multiple vessels bilaterally, which was not surgically remediable.

Intra-arterial guanethidine to the right arm and leg and a chemical sympathectomy to the right leg were successful in resolving the ischaemic changes. Despite the continuation of claudication there has been no further ischaemic ulceration.

\section{CASE 4}

A 48 year old woman was diagnosed as having diffuse scleroderma after presenting with a three month history of Raynaud's phenomenon and progressive scleroderma of the arms, face, and trunk. There was a history of dysphagia and oesophageal disorder was seen on barium swallow. Speckled antinuclear antibodies at a titre of 1/80 were found but antibodies to extractable nuclear antigens were not detected. The only vascular risk factors were a 10 packet-year history of cigarette smoking which stopped 20 years before presentation and a nine year history of mild hypertension. Blood glucose and lipid levels were normal. The activated partial thromboplastin time was not prolonged and anticardiolipin antibodies were not detected. Initial treatment was with D-penicillamine, but after severe gastrointestinal intolerance colchicine $0.5 \mathrm{mg}$ daily was substituted.

At the age of 49 years she developed recurrent ulceration of her fingers which resolved on treatment with nifedipine and antibiotics.

Two years later there was a recurrence of digital ulceration. Digital subtraction angiography showed total occlusion of the two ulnar arteries and severe narrowing of the first digital artery to the right thumb. A left microsympathectomy and ulnar artery resection and bypass procedure were performed and resulted in rapid healing of the ulceration and no recurrence of ischaemia. Haematoxylin and eosin and silver staining of the artery sample showed medial thickening with circumferential luminal narrowing and occlusion by an acellular material. There was no evidence of atherosclerosis as shown by the absence of the characteristic lipid laden macrophages and smooth muscle cells.

\section{Discussion}

Microvascular disease is a well recognised feature of scleroderma manifesting as Raynaud's phenomenon or associated complications including digital infarcts, pulp atrophy, or gangrene. Fixed structural changes in the small vessels are seen in electron microscopic studies of capillaries ${ }^{4}$ and pathological studies of arterioles and small arteries ${ }^{1-3}$ which show concentric intimal proliferation and fibrous adventitial thickening. There is no increase in atherosclerotic disease of large vessels. ${ }^{2}$ Macrovascular disease has been largely unrecognised except for sporadic case reports, ${ }^{5-7}$ all of which were associated with significant morbidity and mortality.

The four patients presented here were all women and developed symptoms of large vessel disease between the ages of 50 and 66 years. Three patients had limited scleroderma and one had diffuse disease, as might be expected from the frequencies of limited versus diffuse disease. There was widespread large vessel disease in patient 3, affecting her arms and legs and possibly the coronary vasculature. In the three patients with CREST syndrome, macrovascular disease was not clinically manifest until more than 10 years after the onset of Raynaud's phenomenon. In the patient with rapidly progressive diffuse scleroderma (patient 4), symptomatic large vessel disease was manifest within three years of the onset of Raynaud's phenomenon, indicating that the progression of macrovascular disease may parallel the skin changes (which may of themselves be manifestations of progressive microvascular disease ${ }^{3}$ ). In only one patient (patient 4) was there any history of known vascular risk factors and even then the hypertension was mild and well controlled and the 10 packet-years smoking history had stopped 21 years before the onset of Raynaud's and 23 years before the onset of clinical macrovascular disease.

A sample from the ulnar artery in patient 4 showed circumferential luminal narrowing and total occlusion by an acellular material. There was medial thickening and silver staining showed fragmentation of the internal elastic lamina. It is of interest that these changes were not those of atherosclerosis, which is characterised by lipid laden macrophages with underlying lipid laden smooth muscle cells. ${ }^{8}$ The lesions were more typical of a severe arteriosclerotic process and resembled the changes seen in small renal vessels in scleroderma of the kidney. The presence of a different mechanism of large vessel obstruction 
would explain the greater than expected severity and unusual distribution of vascular disease in the absence of known risk factors for atherosclerosis. In scleroderma abnormalities of endothelial cell metabolism (perhaps antibody mediated $^{9}$ ) may be responsible for this process, as suggested by abnormalities such as the absence of fibrinolysis at sites of vascular occlusion $^{6}$ and raised levels of factor VIII related antigen. ${ }^{10}$ Our pathological findings suggest that subendothelial migration of monocytes or smooth muscle cells with the associated lipid accumulation so pivotal in the development of atherosclerosis ${ }^{6}$ is not part of this process. The vascular pathology in the amputated legs of patient 1 would have been of great interest, but was not available to us.

Our four patients are from the rheumatology unit of a large city teaching hospital and represent $11 \%$ of the total number of women with scleroderma seen at this hospital since 1974. The prevalence of macrovascular disease is dependent on sex and age and is more common in men, rising in both sexes with increasing age. ${ }^{11-13}$ The reasons why we suspect that macrovascular disease relates not so much to the patients' age as to their scleroderma are twofold. First, population studies suggest that the prevalence of symptomatic vascular disease in women is about $2 \%$, rising to $2 \cdot 7 \%$ in the oldest age groups. ${ }^{11} 12$ Assuming a worst case scenario, the expected number of women with symptomatic vascular disease in our hospital's cohort of women with scleroderma should be $1 \cdot 03$, whereas the observed number of four represents more than a threefold excess above that expected. Second, the pathology showing severe arteriosclerosis rather than the expected age related atherosclerosis is another reason why the association between macrovascular disease and scleroderma appears tenable. Studies are currently underway to test further this apparent association.

1 Norton W L, Nardo J M. Vascular disease in progressive systemic sclerosis (scleroderma). Ann Intern Med 1970 73: $317-24$.

2 D'Angelo W A, Fries J F, Masi A T, Shulman L E. Pathological observations in systemic sclerosis (scleroderma). Am f Med 1969; 46: 428-40.

3 Campbell P M, Leroy E C. Pathogenesis of systemic sclerosis: a vascular hypothesis. Semin Arthritis Rheum 1975; 4: 351-68.

4 Michalowski R, Kudejike J. Electron microscopic observations on skeletal muscle in diffuse scleroderma. $\mathrm{Br} f$ Dermatol 1966; 78: 24-8.

5 Lee J E, Haynes J M. Carotid arteries and cerebral infarction due to scleroderma. Neurology 1967; 17: 18-22.

6 Furey N L, Schmid F R, Kwaan H C, Friederici H H. Arterial thrombosis in scleroderma. Br f Dermatol 1975; 93: 683-93.

7 Dorevitch M I, Clemens L E, Webb J B. Lower limb amputation secondary to large vessel involvement in scleroderma. Br $\mathcal{F}$ Rheumatol 1988; 27: 403-6.

8 Ross R. The pathogenesis of atherosclerosis-an update. N Engl F Med 1986; 314: 488-500.

9 James J P, Stevens R J, Hall N D, et al. Factor VIII related antigen in connective tissue disease patients and relatives. Brf Rheumatol 1990; 29: 6-9.

10 Rosenbaum J R, Pottinger B E, Woo P, Black C M, Byron $M$ A, Pearson J S. Measurement and characterisation of circulating anti-endothelial cell IgG in connective tissue diseases. Clin Exp Immunol 1988; 72: 450-6.

11 Criqui T, Fronek A, Barrett-Connor E, Klauber M R Gabriel S, Goodman D. The prevalence of peripheral arterial disease in a defined population. Circulation 1985; 71: $510-5$.

12 Hughson W G, Mann J I, Garrod A. Intermittent claudication: prevalence and risk factors. BMF 1978; 1: 1379 .

13 Gordon T, Kannel W B. Predisposition to atherosclerosis in the head, heart, and legs. The Framingham Study. $\mathcal{F} A M A$ 1972; 221: 661-6. 